143 DEVELOPMENT OF AN ACUTE INTERVENTION TEAM AN INNOVATIVE SOLUTION TO PROVIDING ENHANCED 24/7 PALLIATIVE AND END OF LIFE SUPPORT IN ACUTE HOSPITALS

Lucy Nicholson, Lisa Ward, Richard Hixon. County Durham and Darlington Foundation Trust (CDDFT)

10.1136/spcare-2020-PCC.163

Background The 'Acute Intervention Team' (AIT) is the culmination of a multi-disciplinary drive to better support parent teams to actively manage deteriorating patients and recognise and care for the patients where palliation is the most appropriate course.

Methods The AIT consists of Nurse Practitioners and Senior Health Care Assistants who, as well as possessing the skills of a typical critical care outreach team, have also received additional dedicated training in palliative and end of life care. This includes a period shadowing the Specialist Palliative Care (SPC) Team and bespoke training on recognition of potential lack of reversibility, diagnosing dying, management of palliative emergencies, symptom control and end of life care.

Results The Team have reviewed over 6000 patients since commencing clinical practice in December 2016. Just under half died during their admission, demonstrating how important palliative care is within the role. Whilst most referrals were for patients with high NEWS, notably 241 were referred by SPC for enhanced palliative support to wards out-of-hours. The percentage of cases where a DNACPR should have been in place for patients suffering a cardiac arrest reduced from $41 \%$ to $30 \%$ in $2017 / 18$, and in $2018 / 19$ reduced further to $17 \%$. This demonstrates the team have had a positive impact on appropriate escalation planning. Palliative care coding has also significantly increased over this time, from $20 \%$ to $40 \%$ of deaths, reflecting improved palliative decision making and quality of care.

Conclusion This innovative team are addressing the challenges of providing good 24/7 end-of-life care for the approximately 2000 patients who die each year within CDDFT, allowing us to better meet the challenges of modern day healthcare. Proactive care for these patients now includes escalation to critical care or to palliative care. Replication of this model would be cost neutral for any trust with a critical care outreach team.

\section{THE 'TECHNOLOGY IN PALLIATIVE CARE' (TIP) STUDY: A DELPHI STUDY TO DEFINE RESEARCH PRIORITIES FOR THE USE OF TECHNOLOGY FOR THE CLINICAL MANAGEMENT OF PEOPLE WITH SERIOUS ILLNESS}

Amara C Nwosu, Tamsin McGlinchey, Stephen Mason. Palliative Care Institute Liverpool, University of Liverpool, Marie Curie Hospice Liverpool

\subsection{6/spcare-2020-PCC.164}

Background The development of data-driven technology presents an opportunity to improve quality and efficiency of healthcare provision and patient experience. It is important to carefully evaluate current and planned future developments, to identify research priority areas and determine how resources should be best utilised. This project aimed to explore how digital technology can improve the care of people with palliative care needs by setting 'priority areas' for further research and development.

Methods and objectives A scoping literature review was conducted. Two rounds of international Delphi questionnaires were completed, followed by a consensus meeting of experts and a public engagement event.

Results One hundred and eight experts (from healthcare, academic and technology) from 11 countries participated in round 1 Delphi. The second round Delphi questionnaire was completed by 55 (53\% of 1 st round) participants in nine countries. Eleven experts attended the final consensus meeting. A total of 12 priorities were selected (from a list of 33) which were summarised into eight themes. These themes were: Big Data; mobile devices; ehealth/telehealth; virtual reality; smart homes; biotechnology and digital legacy. A public engagement meeting of six lay representative was conducted to discuss each priority area in depth and identify future research questions.

Conclusion The identified priorities represent a wide range of important emerging areas in field of digital health, personalised medicine and data science. Collaborative studies are required to address these priority areas, which will aim to improve the care and experience for people with palliative care needs.

\section{PALLIATIVE CARE OUTCOMES COLLABORATION ASSESSMENT TOOLS IN PRACTICE: A SET OF 4 AUDITS}

Frances O'Mahony, Siobhan Coffey, Claire Kearns, John Sheridan, Sue Moran, Martina O'Reilly. Milford Care Centre, Limerick

\subsection{6/spcare-2020-PCC. 165}

Background PCOC uses a suite of clinical assessment tools to help identify and manage common symptoms and problems among palliative care patients. They may help patients, carers and families to communicate their experiences and preferences with clinicians and, importantly, to benchmark outcomes. PCOC assessment tools are being adopted widely in the Irish palliative care setting at the moment.

Methods PCOC assessments were introduced in the inpatient specialist palliative care unit at Milford Care Centre in December 2018. 4 separate audits were conducted during this initial period: Phase Definition, Phase Change, Phase Assessment, Problem Severity Score (PSS) Assessment.

Results Phase Definition and Phase Change: Of a total of 127 phases among 10 patients, 91 (71.65\%) were correct, $21(16.54 \%)$ were incorrect and 15 (11.81\%) were missed. Most errors related to recognition of the terminal phase and recording of a change of phase outside of routine assessment times. Phase and PSS Assessment: These examined the consistency of scores applied by two different clinicians when assessing a patient within two hours. Initially, our compliance rates were approaching those published previously. Following full implementation of training for all clinical staff, a re-audit showed the rate of consistency matched the published rates for all domains except for assessment of 'other symptoms' (aside from pain) and 'family/carer problems.' The latter improved significantly in the period between data collection.

Conclusions Overall results are very positive and areas for improvement have been identified. Education and dissemination of results to clinical staff is continuing. A positive response to 